\title{
Global Stability of Zika Virus Dynamics
}

\author{
Savannah Bates \\ Hayley Hutson \\ MSU Undergraduate \\ Jorge Rebaza \\ Missouri State University
}

Follow this and additional works at: https://bearworks.missouristate.edu/articles-cnas

\section{Recommended Citation}

Bates, Savannah, Hayley Hutson, and Jorge Rebaza. "Global stability of Zika virus dynamics." Differential Equations and Dynamical Systems (2017): 1-16.

This article or document was made available through BearWorks, the institutional repository of Missouri State University. The work contained in it may be protected by copyright and require permission of the copyright holder for reuse or redistribution.

For more information, please contact BearWorks@library.missouristate.edu. 
K. Bessey, M. Mavis, J. Rebaza*, and J. Zhang

\title{
Global Stability Analysis of a General Model of Zika Virus
}

https://doi.org/10.1515/msds-2019-0002

Received December 4, 2018; accepted March 21, 2019

\begin{abstract}
Mathematical models of Zika virus dynamics are relatively new, and they mostly focus on either vector and horizontal, or vector and vertical transmission only. In this work, we first revisit a recent model that considers vector and vertical transmission, and we provide an alternative proof on the global stability of the disease-free equilibrium point. Then, a new and general model is presented which includes vector, horizontal and vertical transmission. For this new model, existence of both a disease-free and an endemic equilibrium is studied. Using matrix and graph-theoretic methods, appropriate Lyapunov functions are constructed and results on the global stability properties of both equilibria are established.
\end{abstract}

Keywords: Disease epidemics, dynamical systems, global stability, Lyapunov functions

MSC: 37N25, 92D25

\section{Introduction}

Zika virus is mainly transmitted to humans through bites of infected female mosquitoes from the Aedes genus. Infected humans can pass this disease to other humans via horizontal (sexual activity) or vertical (motherchild) transmission. In addition, infected humans can also pass the disease to susceptible mosquitoes, when bitten. In general, the stage of the infection determines the type and the intensity of the transmission: during the incubation period, infected humans can infect both susceptible mosquitoes and humans; after this exposed period, symptomatic humans are more contagious to mosquitoes, and at the convalescence stage, they can no longer infect mosquitoes, but they can still spread the infection to humans through sexual activity. Some studies suggest a correlation between Zika virus infection and congenital anomalies such as microcephaly and Guillain-Barre syndrome. Data available for these studies mostly come from the outbreaks in Micronesia (2007), Polynesia (2013), and Brazil (2015). We refer the reader to $[1,4,8,10,13,14,20]$ and the references therein for a detailed biological and epidemiological description of the Zika virus.

Various types of disease epidemics have been extensively studied mathematically (e.g. $[6,11,12,16,18,19]$ ). Mathematical modeling of these diseases has helped understand the dynamics of the disease, the conditions under which an outbreak can occur, and how the disease would spread. However, mathematical models on the dynamics of Zika virus are relatively new, and most of them study vector (mosquito-human and humanmosquito) and horizontal transmission only [2, 10, 14, 17]. Evidence of Zika virus vertical (congenital and perinatal) transmission has been reported by the Center for Disease Control (CDC). Further possible evidence of vertical transmission has only recently been reported (e.g. [3, 5, 13, 20]). A recent work [1], considered a mathematical model for vector and vertical Zika transmission, but did not include horizontal transmission;

K. Bessey: Department of Mathematics, University of North Georgia, E-mail: khbess2478@ung.edu

M. Mavis: Rosenstiel School of Marine and Atmospheric Science, University of Miami, E-mail: mem126@miami.edu

*Corresponding Author: J. Rebaza: Department of Mathematics, Missouri State University, E-mail: jrebaza@missouristate.edu J. Zhang: Department of Mathematics, Johns Hopkins University, E-mail: jzhan@154@jhu.edu 
the authors also studied the development of microcephaly in newborns, and identified the most important parameters that influence the spread of the disease.

In this work, we extend and generalize previous research on mathematical models for Zika virus dynamics by proposing a model that includes vector, horizontal and vertical transmission simultaneously. While for most models on Zika virus (e.g. [1, 10, 17]), an endemic equilibrium does not exist, the dynamical system in this new model has both a disease-free and an endemic equilibrium, and we provide rigorous proofs on the global stability properties of both equilibrium points.

We start by revisiting the model presented in [1], and provide an alternative proof on the global stability of the disease-free equilibrium, under fewer restrictions. We then propose a new and more general model that includes vector, horizontal and vertical transmission. Using matrix and graph-theoretic methods from [19], we provide results on the global stability properties of both equilibria. To the best of our knowledge, this is the first model of Zika virus dynamics that includes the three types of transmission.

\section{Vector and Vertical Transmission}

\subsection{The Original Model}

We start by studying the model presented by Agusto et al. [1], which includes vector and vertical transmission only. Human population is divided into newborns, denoted with a $B$ subscript, and everyone else (referred to as adults), denoted with a $W$ subscript. An individual with microcephaly is denoted with an $M$ subscript. Table 1 describes in detail the variables and parameters under study. The model proposed in [1] is given by:

$$
\begin{aligned}
S_{B}^{\prime} & =\pi_{B}-q_{A} \pi_{B} A_{W}-q_{I} \pi_{B} I_{W}-q_{R} \pi_{B} R_{W}-\lambda_{B}\left(I_{V}, N_{B}\right) S_{B}-\left(\alpha+\mu_{B}\right) S_{B} \\
E_{B}^{\prime} & =\lambda_{B}\left(I_{V}, N_{B}\right) S_{B}-\left(\alpha+\sigma_{B}+\mu_{B}\right) E_{B} \\
A_{B}^{\prime} & =q_{A} \pi_{B} A_{W}+(1-p) \sigma_{B} E_{B}-\left(\alpha+\gamma_{B}+\mu_{B}\right) A_{B} \\
I_{B}^{\prime} & =q_{I} \pi_{B} I_{W}+p \sigma_{B} E_{B}-\left(\alpha+\gamma_{B}+\mu_{B}\right) I_{B} \\
I_{B M}^{\prime} & =r q_{R} \pi_{B} R_{W}-\left(\alpha+\mu_{B}\right) I_{B M} \\
R_{B}^{\prime} & =(1-r) q_{R} \pi_{B} R_{W}+\gamma_{B} A_{B}+\gamma_{B} I_{B}-\left(\alpha+\mu_{B}\right) R_{B} \\
S_{W}^{\prime} & =\alpha S_{B}-\lambda_{W}\left(I_{V}, N_{W}\right) S_{W}-\mu_{W} S_{W} \\
E_{W}^{\prime} & =\lambda_{W}\left(I_{V}, N_{W}\right) S_{W}-\left(\sigma_{W}+\mu_{W}\right) E_{W} \\
A_{W}^{\prime} & =(1-p) \sigma_{W} E_{W}-\left(\gamma_{W}+\mu_{W}\right) A_{W} \\
I_{W}^{\prime} & =p \sigma_{W} E_{W}-\left(\gamma_{W}+\mu_{W}\right) I_{W} \\
I_{W M}^{\prime} & =\alpha I_{B M}-\mu_{W} I_{W M} \\
R_{W}^{\prime} & =\alpha R_{B}+\gamma_{W} A_{W}+\gamma_{W} I_{W}-\mu_{W} R_{W} \\
S_{V}^{\prime} & =\pi_{V}-\lambda_{V}\left(A_{B}, I_{B}, A_{W}, I_{W}, N_{B}, N_{W}\right) S_{V}-\mu_{V} S_{V} \\
E_{V}^{\prime} & =\lambda_{V}\left(A_{B}, I_{B}, A_{W}, I_{W}, N_{B}, N_{W}\right) S_{V}-\left(\mu_{V}+\sigma_{V}\right) E_{V} \\
I_{V}^{\prime} & =\sigma_{V} E_{V}-\mu_{V} I_{V},
\end{aligned}
$$

where $\lambda_{W}\left(I_{V}, N_{W}\right)=\frac{\beta_{W} b_{V} I_{V}}{N_{W}}, \quad \lambda_{B}\left(I_{V}, N_{B}\right)=\frac{\eta \beta_{B} b_{V} I_{V}}{N_{B}}$, $\lambda_{V}\left(A_{B}, I_{B}, A_{W}, I_{W}, N_{B}, N_{W}\right)=\beta_{V} b_{V}\left(\frac{I_{W}+\rho_{W} A_{W}+\eta\left(I_{B}+\rho_{B} A_{B}\right)}{N_{W}+\eta N_{B}}\right)$, and (') denotes derivative with respect to $t$. We always assume $t \geq 0$.

The total populations are given by:

$$
\begin{aligned}
N_{B} & =S_{B}+E_{B}+A_{B}+I_{B}+I_{B M}+R_{B} \\
N_{W} & =S_{W}+E_{W}+A_{W}+I_{W}+I_{W M}+R_{W} \\
N_{V} & =S_{V}+E_{V}+I_{V},
\end{aligned}
$$

and $N_{H}=N_{B}+N_{W}$. All variables are non-negative, and all parameters are strictly positive (except for $\rho_{B}, \rho_{W}$, which are taken to be non-negative). 
Table 1: Adapted from Table 1 in [1].

\begin{tabular}{l|l}
\hline Variable & Description \\
\hline$S_{B}, S_{W}$ & Susceptible newborns and adults \\
$E_{B}, E_{W}$ & Exposed newborns and adults \\
$A_{B}, A_{W}$ & Asymptomatic newborns and adults \\
$I_{B}, I_{W}$ & Symptomatic newborns and adults without microcephaly \\
$I_{B M}, I_{W M}$ & Newborns and adults with microcephaly \\
$R_{B}, R_{W}$ & Recovered newborns and adults \\
$S_{V}, E_{V}, I_{V}$ & Susceptible, exposed and infected female mosquitoes \\
\hline Parameter & Description \\
\hline$\pi_{B}$ & Birth rate \\
$p$ & Fraction of newborns and adults who are infectious \\
$1-p$ & Remaining fraction of adults and newborns who are asymptomatic \\
$\alpha$ & Maturation rate \\
$r, q_{A}, q_{I}, q_{R}$ & Fractions of newborns with microcephaly, or vertically infected \\
$1-r$ & Remaining fraction of newborns who are recovered \\
$\beta_{B}, \beta_{W}$ & Transmission probability per contact of newborns and adults \\
$\eta, \rho_{B}, \rho_{W}$ & Modification parameters \\
$\sigma_{B}, \sigma_{W}$ & Progression rate of exposed newborns and adults \\
$\gamma_{B}, \gamma_{W}$ & Recovery rate of newborns and adults \\
$\mu_{B}, \mu_{W}$ & Natural death rate of newborns and adults \\
$\pi_{V}$ & Recruitment rate of mosquitoes \\
$\beta_{V}$ & Transmission probability per contact of susceptible mosquitoes \\
$b_{V}$ & Mosquito biting rate \\
$\sigma_{V}$ & Progression rate of exposed mosquitoes \\
$\mu_{V}$ & Natural death rate of mosquitoes \\
\hline
\end{tabular}

The terms $q_{A} \pi_{B} A_{W}, q_{I} \pi_{B} I_{W}, q_{R} \pi_{B} R_{W}$ in model (2.1) represent the vertical (mother - child) transmission, the functions $\lambda_{B}$ and $\lambda_{W}$ represent vector transmission from mosquitoes to newborns and adults respectively, and the function $\lambda_{V}$ represents vector transmission from humans to mosquitoes. According to this model, asymptomatic and symptomatic mothers can give birth to asymptomatic or symptomatic babies respectively, while recovered mothers can give birth to babies who either have microcephaly or are recovered. On the other hand, humans exposed to the disease through vector transmission progress to either asymptomatic or symptomatic stages. It is also assumed that Zika virus generates lifelong immunity but individuals with microcephaly have a short lifespan. An SEI-type model is assumed for mosquitoes. For a more detailed interpretation of this model, see [1].

For model (2.1), one can readily show that the feasible region is

$$
\Gamma_{1}=\Gamma_{H} \times \Gamma_{V} \subseteq \mathbb{R}_{+}^{12} \times \mathbb{R}_{+}^{3},
$$

where

$$
\begin{gathered}
\Gamma_{H}=\left\{\left(S_{B}, E_{B}, A_{B}, I_{B}, I_{B M}, R_{B}, S_{W}, E_{W}, A_{W}, I_{W}, I_{W M}, R_{W}\right) \in \mathbb{R}_{+}^{12}: N_{H} \leq \frac{\pi_{B}}{\mu_{H}}+N_{H}(0)\right\}, \\
\text { and } \quad \Gamma_{V}=\left\{\left(S_{V}, E_{V}, I_{V}\right) \in \mathbb{R}_{+}^{3}: N_{V} \leq \frac{\pi_{V}}{\mu_{V}}+N_{V}(0)\right\},
\end{gathered}
$$

with $\mu_{H}=\min \left\{\mu_{B}, \mu_{W}\right\} . \Gamma_{1}$ is compact and invariant with respect to (2.1). 
Remark 1. Note that the sets $\Gamma_{H}$ and $\Gamma_{V}$ here are different than the ones given in [1].

System (2.1) has only one equilibrium point, which corresponds to the disease-free state. This disease-free equilibrium (DFE), denoted as $E^{0}=\left(S_{B}^{0}, E_{B}^{0}, A_{B}^{0}, I_{B}^{0}, I_{B M}^{0}, R_{B}^{0}, S_{W}^{0}, E_{W}^{0}, A_{W}^{0}, I_{W}^{0}, I_{W M}^{0}, R_{W}^{0}, S_{V}^{0}, E_{V}^{0}, I_{V}^{0}\right)$ is given by:

$$
E^{0}=\left(\frac{\pi_{B}}{\alpha+\mu_{B}}, 0,0,0,0,0, \frac{\alpha \pi_{B}}{\mu_{W}\left(\alpha+\mu_{B}\right)}, 0,0,0,0,0, \frac{\pi_{V}}{\mu_{V}}, 0,0\right) .
$$

No endemic equilibrium point exists for this system.

\subsection{Global Asymptotic Stability Analysis of the DFE}

To analyze the global asymptotic stability of the DFE, we consider system (2.1) in compartmental form [19, 21], by splitting the variables into two compartments: a disease compartment $x \in \mathbb{R}^{10}$ and a nondisease compartment $y \in \mathbb{R}^{5}$ :

$$
\begin{gathered}
x=\left[E_{B}, A_{B}, I_{B}, I_{B M}, E_{W}, A_{W}, I_{W}, I_{W M}, E_{V}, I_{V}\right]^{T} \text {, and } \\
y=\left[S_{B}, R_{B}, S_{W}, R_{W}, S_{V}\right] .^{T}
\end{gathered}
$$

The entries in vectors $\mathcal{F}(x, y)$ and $\mathcal{V}(x, y) \in \mathbb{R}^{10}$ below, represent rate of new infections and transition terms respectively in the $i$-th disease compartment:

$$
\mathcal{F}=\left[\begin{array}{c}
\frac{\eta \beta_{B} b_{V} I_{V}}{N_{B}} S_{B} \\
0 \\
0 \\
r q_{R} \pi_{B} R_{W} \\
\frac{\beta{ }_{W} b_{V} I_{V}}{N_{W}} S_{W} \\
0 \\
0 \\
0 \\
U \\
0
\end{array}\right], \quad \mathcal{N}=\left[\begin{array}{c}
\left(\alpha+\sigma_{B}+\mu_{B}\right) E_{B} \\
\left(\alpha+\gamma_{B}+\mu_{B}\right) A_{B}-q_{A} \pi_{B} A_{W}-(1-p) \sigma_{B} E_{B} \\
\left(\alpha+\gamma_{B}+\mu_{B}\right) I_{B}-q_{I} \pi_{B} I_{W}-p \sigma_{B} E_{B} \\
\left(\alpha+\mu_{B}\right) I_{B M} \\
\left(\sigma_{W}+\mu_{W}\right) E_{W} \\
\left(\gamma_{W}+\mu_{W}\right) A_{W}-(1-p) \sigma_{W} E_{W} \\
\left(\gamma_{W}+\mu_{W}\right) I_{W}-p \sigma_{W} E_{W} \\
\mu_{W} I_{W M}-\alpha I_{B M} \\
\left(\mu_{V}+\sigma_{V}\right) E_{V} \\
\mu_{V} I_{V}-\sigma_{V} E_{V}
\end{array}\right],
$$

where $U=\beta_{V} b_{V}\left(\frac{I_{W}+\rho_{W} A_{W}+\eta\left(I_{B}+\rho_{B} A_{B}\right)}{N_{W}+\eta N_{B}}\right) S_{V}$.

Note that the assumptions in [19]: $\mathcal{F}_{i}(0, y)=0, \mathcal{V}_{i}(0, y)=0, \mathcal{F}_{i}(x, y) \geq 0, \mathcal{V}_{i}(x, y) \leq 0$ when $x_{i}=0$, and $\sum_{i=1}^{10} \mathcal{V}_{i}(x, y) \geq 0$ for $i=1, \ldots, 10$ hold. We also define:

$$
F=\left[\frac{\partial \mathcal{F}_{i}}{\partial x_{j}}\left(0, y_{0}\right)\right], \text { and } V=\left[\frac{\partial \mathcal{V}_{i}}{\partial x_{j}}\left(0, y_{0}\right)\right] \text {, }
$$


where $y_{0}=\left(\frac{\pi_{B}}{\alpha+\mu_{B}}, 0, \frac{\alpha \pi_{B}}{\mu_{W}\left(\alpha+\mu_{B}\right)}, 0, \frac{\pi_{V}}{\mu_{V}}\right)$. This gives

$$
\begin{aligned}
& F=\left[\begin{array}{cccccccccc}
0 & 0 & 0 & 0 & 0 & 0 & 0 & 0 & 0 & \eta \beta_{B} b_{V} \\
0 & 0 & 0 & 0 & 0 & 0 & 0 & 0 & 0 & 0 \\
0 & 0 & 0 & 0 & 0 & 0 & 0 & 0 & 0 & 0 \\
0 & 0 & 0 & 0 & 0 & 0 & 0 & 0 & 0 & 0 \\
0 & 0 & 0 & 0 & 0 & 0 & 0 & 0 & 0 & \beta_{W} b_{V} \\
0 & 0 & 0 & 0 & 0 & 0 & 0 & 0 & 0 & 0 \\
0 & 0 & 0 & 0 & 0 & 0 & 0 & 0 & 0 & 0 \\
0 & 0 & 0 & 0 & 0 & 0 & 0 & 0 & 0 & 0 \\
0 & \frac{\eta \beta_{V} b_{V} \rho_{B} S_{V}^{0}}{S_{W}^{0}+\eta S_{B}^{0}} & \frac{\eta \beta_{V} b_{V} S_{V}^{0}}{S_{W}^{0}+\eta S_{B}^{0}} & 0 & 0 & \frac{\rho_{W} \beta_{V} b_{V} S_{V}^{0}}{S_{W}^{0}+\eta S_{B}^{0}} & \frac{\beta_{V} b_{V} S_{V}^{0}}{S_{W}^{0}+\eta S_{B}^{0}} & 0 & 0 & 0 \\
0 & 0 & 0 & 0 & 0 & 0 & 0 & 0 & 0 & 0
\end{array}\right], \\
& V=\left[\begin{array}{cccccccccc}
k_{1} & 0 & 0 & 0 & 0 & 0 & 0 & 0 & 0 & 0 \\
-(1-p) \sigma_{B} & k_{2} & 0 & 0 & 0 & -q_{A} \pi_{B} & 0 & 0 & 0 & 0 \\
-p \sigma_{B} & 0 & k_{2} & 0 & 0 & 0 & -q_{I} \pi_{B} & 0 & 0 & 0 \\
0 & 0 & 0 & k_{3} & 0 & 0 & 0 & 0 & 0 & 0 \\
0 & 0 & 0 & 0 & k_{4} & 0 & 0 & 0 & 0 & 0 \\
0 & 0 & 0 & 0 & -(1-p) \sigma_{W} & k_{5} & 0 & 0 & 0 & 0 \\
0 & 0 & 0 & 0 & -p \sigma_{W} & 0 & k_{5} & 0 & 0 & 0 \\
0 & 0 & 0 & -\alpha & 0 & 0 & 0 & \mu_{W} & 0 & 0 \\
0 & 0 & 0 & 0 & 0 & 0 & 0 & 0 & k_{6} & 0 \\
0 & 0 & 0 & 0 & 0 & 0 & 0 & 0 & -\sigma_{V} & \mu_{V}
\end{array}\right] \text {, }
\end{aligned}
$$

where $k_{1}=\alpha+\sigma_{B}+\mu_{B}, k_{2}=\alpha+\gamma_{B}+\mu_{B}, k_{3}=\alpha+\mu_{B}, k_{4}=\sigma_{W}+\mu_{W}, k_{5}=\gamma_{W}+\mu_{W}, k_{6}=\mu_{V}+\sigma_{V}$. The next generation matrix (NGM) $[19,21]$ is:

$$
F V^{-1}=\left[\begin{array}{cccccccccc}
0 & 0 & 0 & 0 & 0 & 0 & 0 & 0 & A & B \\
0 & 0 & 0 & 0 & 0 & 0 & 0 & 0 & 0 & 0 \\
0 & 0 & 0 & 0 & 0 & 0 & 0 & 0 & 0 & 0 \\
0 & 0 & 0 & 0 & 0 & 0 & 0 & 0 & 0 & 0 \\
0 & 0 & 0 & 0 & 0 & 0 & 0 & 0 & C & D \\
0 & 0 & 0 & 0 & 0 & 0 & 0 & 0 & 0 & 0 \\
0 & 0 & 0 & 0 & 0 & 0 & 0 & 0 & 0 & 0 \\
0 & 0 & 0 & 0 & 0 & 0 & 0 & 0 & 0 & 0 \\
E & G & H & 0 & I & J & K & 0 & 0 & 0 \\
0 & 0 & 0 & 0 & 0 & 0 & 0 & 0 & 0 & 0
\end{array}\right],
$$


with

$$
\begin{aligned}
A= & \frac{\sigma_{V} \eta \beta_{B} b_{V}}{\left(\mu_{V}+\sigma_{V}\right) \mu_{V}}, B=\frac{\eta \beta_{B} b_{V}}{\mu_{V}}, C=\frac{\sigma_{V} \beta_{W} b_{V}}{\left(\mu_{V}+\sigma_{V}\right) \mu_{V}}, D=\frac{\beta_{W} b_{V}}{\mu_{V}}, \\
E= & \frac{\sigma_{B} \eta \beta_{V} b_{V} S_{V}^{0}\left[(1-p) \rho_{B}+p\right]}{\left(\alpha+\sigma_{B}+\mu_{B}\right)\left(\alpha+\gamma_{B}+\mu_{B}\right)\left(S_{W}^{0}+\eta S_{B}^{0}\right)}, \\
G= & \frac{\eta \rho_{B} \beta_{V} b_{V} S_{V}^{0}}{\left(\alpha+\gamma_{B}+\mu_{B}\right)\left(S_{W}^{0}+\eta S_{B}^{0}\right)}, H=\frac{\eta \beta_{V} b_{V} S_{V}^{0}}{\left(\alpha+\gamma_{B}+\mu_{B}\right)\left(S_{W}^{0}+\eta S_{B}^{0}\right)}, \\
I= & \frac{\sigma_{W} \beta_{V} b_{V} S_{V}^{0}\left[(1-p) \rho_{W}+p\right]}{\left(\sigma_{W}+\mu_{W}\right)\left(\gamma_{W}+\mu_{W}\right)\left(S_{W}^{0}+\eta S_{B}^{0}\right)}+ \\
& \frac{\pi_{B} \sigma_{W} \eta \beta_{V} b_{V} S_{V}^{0}\left[q_{I} p+q_{A}(1-p) \rho_{B}\right]}{\left(\sigma_{W}+\mu_{W}\right)\left(\gamma_{W}+\mu_{W}\right)\left(\alpha+\mu_{B}+\gamma_{B}\right)\left(S_{W}^{0}+\eta S_{B}^{0}\right)}, \\
J= & \frac{\beta_{V} b_{V} S_{V}^{0}}{\left(\gamma_{W}+\mu_{W}\right)\left(S_{W}^{0}+\eta S_{B}^{0}\right)}\left[\rho_{W}+\frac{q_{A} \pi_{B} \eta \rho_{B}}{\left(\alpha+\gamma_{B}+\mu_{B}\right)}\right], \\
K= & \frac{\beta_{V} b_{V} S_{V}^{0}}{\left(\gamma_{W}+\mu_{W}\right)\left(S_{W}^{0}+\eta S_{B}^{0}\right)}\left[1+\frac{q_{I} \pi_{B} \eta}{\left(\alpha+\gamma_{B}+\mu_{B}\right)}\right] .
\end{aligned}
$$

The basic reproduction number $\mathcal{R}_{0}$, defined as the expected number of secondary cases produced by an infected individual in a completely susceptible population $[7,21]$, is the spectral radius $\rho$ of the NGM:

$$
\mathcal{R}_{0}=\rho\left(F V^{-1}\right)=\sqrt{A E+C I},
$$

where $A, C, E$, and $I$ are defined as above. $\mathcal{R}_{0}$ in (2.5) is actually an eigenvalue of the matrix $F V^{-1}$, computed directly using the characteristic equation of (2.4).

Remark 2. The basic reproduction number in (2.5) can be written as

$$
\mathcal{R}_{0}=\sqrt{\mathcal{R}_{V}\left(\mathcal{R}_{B}+\mathcal{R}_{\mathcal{W}}\right)}
$$

with $\mathcal{R}_{V}=\frac{\beta_{V} b_{V} \sigma_{V} S_{V}^{0}}{k_{6} \mu_{V}}, \quad \mathcal{R}_{B}=\frac{\eta^{2} \beta_{B} b_{V} \sigma_{B}\left[(1-p) \rho_{B}+p\right]}{k_{1} k_{2}\left(S_{W}^{0}+\eta S_{B}^{0}\right)}$, and

$\mathcal{R}_{W}=\frac{\beta_{W} b_{V} \sigma_{W}}{k_{2} k_{4} k_{5}\left(S_{W}^{0}+\eta S_{B}^{0}\right)}\left[(1-p)\left(k_{2} \rho_{W}+\eta \rho_{B} q_{A} \pi_{B}\right)+p\left(k_{2}+\eta q_{I} \pi_{B}\right)\right]$.

See [1] for biological interpretations of $\mathcal{R}_{V}, \mathcal{R}_{B}$ and $\mathcal{R}_{\mathcal{W}}$.

In the theorem below, we make the assumption $S_{V} \leq S_{V}^{0}$, which is biologically reasonable.

Theorem 1. If $\mathcal{R}_{0} \leq 1$, then the disease-free equilibrium point $E^{0}$ (2.2) is globally asymptotically stable (GAS) in $\Gamma_{1}$.

Proof. Following the matrix-theoretic method in [19], we set

$$
f(x, y):=(F-V) x-\mathcal{F}(x, y)+\mathcal{V}(x, y),
$$

which gives

$$
f(x, y)=\left[\begin{array}{c}
\eta \beta_{B} b_{V} I_{V}\left(\frac{N_{B}-S_{B}}{N_{B}}\right) \\
0 \\
0 \\
-r q_{R} \pi_{B} R_{W} \\
\beta_{W} b_{V} I_{V}\left(\frac{N_{W}-S_{W}}{N_{W}}\right) \\
0 \\
0 \\
0 \\
\beta_{V} b_{V}\left(I_{W}+\rho_{W} A_{W}+\eta\left(I_{B}+\rho_{B} A_{B}\right)\right)\left(\frac{S_{V}^{0}}{S_{W}^{0}+\eta S_{B}^{0}}-\frac{S_{V}}{N_{W}+\eta N_{B}}\right) \\
0
\end{array}\right] .
$$


Note that we are not able to use Theorem 2.1 in [19] directly, as the condition $f(x, y) \geq 0$ fails (only on the fourth entry). However, a Lyapunov function in the form proposed by the authors can still be constructed. Indeed, define the function

$$
Q=\omega^{T} V^{-1} x,
$$

where $\omega$ is a left eigenvector of the matrix $V^{-1} F$ corresponding to the eigenvalue $\mathcal{R}_{0}$. We are not able to use Theorem 2.2 in [19] either, as the matrix $V^{-1} F$ is not irreducible. However, one can compute a nonnegative left eigenvector $\omega$ of $V^{-1} F$, corresponding to $\mathcal{R}_{0}$. In fact, the eigenvector has the form

$$
\omega^{T}=\left[\begin{array}{llllllllll}
0 & a & b & 0 & 0 & c & d & 0 & 0 & e
\end{array}\right]
$$

where $a, b, c, d$, and $e$ are positive values. One can readily verify that $V^{-1}$ is nonnegative, and hence $Q(x) \geq 0$. Also observe that $Q(x)=0$ at the DFE. We then have

$$
Q^{\prime}=\omega^{T} V^{-1} x^{\prime}=\omega^{T} V^{-1}(F-V) x-\omega^{T} V^{-1} f(x, y)=\left(\mathcal{R}_{0}-1\right) \omega^{T} x-\omega^{T} V^{-1} f(x, y) .
$$

Even though the condition $f(x, y) \geq 0$ is not satisfied, one can verify that the product $\omega^{T} V^{-1} f(x, y)$ is nonnegative. Thus, the condition $\mathcal{R}_{0} \leq 1$ implies $Q^{\prime}=\left(\mathcal{R}_{0}-1\right) \omega^{T} x-\omega^{T} V^{-1} f(x, y) \leq 0$. Therefore, $Q$ is in fact a Lyapunov function in $\Gamma_{1}$.

To prove global stability, first consider $\mathcal{R}_{0}<1$, and let $S=\left\{z \in \mathbb{R}_{+}^{15}: Q^{\prime}=0\right\}$. When $Q^{\prime}=0$, we must have that $\left(\mathcal{R}_{0}-1\right) \omega^{T} x=\omega^{T} V^{-1} f(x, y)$. Since $\mathcal{R}_{0}<1$, we have $\left(\mathcal{R}_{0}-1\right) \omega^{T} x$ non-positive but $\omega^{T} V^{-1} f(x, y)$ non-negative. Thus, $\left(\mathcal{R}_{0}-1\right) \omega^{T} x=0$, and hence $w^{T} x=0$. This implies that $A_{B}=I_{B}=A_{W}=I_{W}=I_{V}=0$, and we get $S=\left\{z \in \mathbb{R}_{+}^{15}: A_{B}=I_{B}=A_{W}=I_{W}=I_{V}=0\right\}$. On this set $S$, we are left with the following system:

$$
\begin{aligned}
S_{B}^{\prime} & =\pi_{B}-q_{R} \pi_{B} R_{W}-\left(\alpha+\mu_{B}\right) S_{B} \\
E_{B}^{\prime} & =-\left(\alpha+\sigma_{B}+\mu_{B}\right) E_{B} \\
I_{B M}^{\prime} & =r q_{R} \pi_{B} R_{W}-\left(\alpha+\mu_{B}\right) I_{B M} \\
R_{B}^{\prime} & =(1-r) q_{R} \pi_{B} R_{W}-\left(\alpha+\mu_{B}\right) R_{B} \\
S_{W}^{\prime} & =\alpha S_{B}-\mu_{W} S_{W} \\
E_{W}^{\prime} & =-\left(\sigma_{W}+\mu_{W}\right) E_{W} \\
I_{W M}^{\prime} & =\alpha I_{B M}(t)-\mu_{W} I_{W M} \\
R_{W}^{\prime} & =\alpha R_{B}(t)-\mu_{W} R_{W} \\
S_{V}^{\prime} & =\pi_{V}-\mu_{v} S_{V} \\
E_{V}^{\prime} & =-\left(\mu_{V}+\sigma_{V}\right) E_{V} .
\end{aligned}
$$

Note that $\omega^{T} x=0$ does not imply $x=0$ (which would lead to the disease-free system as in the proof of Theorem 2.2 in [19] ), because $w$ is not strictly positive. However, one can show that system (2.7) has the unique equilibrium point - see notation in (2.2):

$$
\left(S_{B}^{0}, E_{B}^{0}, I_{B M}^{0}, R_{B}^{0}, S_{W}^{0}, E_{W}^{0}, I_{W M}^{0}, R_{W}^{0}, S_{V}^{0}, E_{V}^{0}\right),
$$

and that this point is GAS for this system. On this set $S$, we also have

$$
A_{B}=A_{B}^{0}, I_{B}=I_{B}^{0}, A_{W}=A_{W}^{0}, I_{W}=I_{W}^{0}, I_{V}=I_{V}^{0} .
$$

Thus, when $\mathcal{R}_{0}<1$, the largest and only invariant set where $Q^{\prime}=0$ is the DFE.

Now let $\mathcal{R}_{0}=1$. Then, from $Q^{\prime}=0$ we get $\omega^{T} V^{-1} f(x, y)=0$. One can show that this implies $S_{B}=N_{B}$ and $S_{W}=N_{W}$, and therefore $E_{B}=A_{B}=I_{B}=I_{B M}=R_{B}=0$, and $E_{W}=A_{W}=I_{W}=I_{W M}=R_{W}=0$. Thus, on this new set $\tilde{S}$ where $Q^{\prime}=0$, we are left with the system

$$
\begin{aligned}
S_{B}^{\prime} & =\pi_{B}-\eta \beta_{B} b_{V} I_{V}-\left(\alpha+\mu_{B}\right) S_{B} \\
S_{W}^{\prime} & =\alpha S_{B}-\beta_{W} b_{v} I_{V}-\mu_{W} S_{W} \\
S_{V}^{\prime} & =\pi_{V}-\mu_{V} S_{V} \\
E_{V}^{\prime} & =-\left(\mu_{V}+\sigma_{V}\right) E_{V} \\
I_{V}^{\prime} & =\sigma_{V} E_{V}-\mu_{V} I_{V} .
\end{aligned}
$$


System (2.8) has the unique equilibrium point: $\left(S_{B}^{0}, S_{W}^{0}, S_{V}^{0}, E_{V}^{0}, I_{V}^{0}\right)$ - with notation as given in (2.2) - which is GAS for this system. On this set $\tilde{S}$, we also have $E_{B}=E_{B}^{0}, A_{B}=A_{B}^{0}, I_{B}=I_{B}^{0}, I_{B M}=I_{B M}^{0}, R_{B}=R_{B}^{0}$, and $E_{W}=E_{W}^{0}, A_{W}=A_{B}^{0}, I_{W}=I_{W}^{0}, I_{W M}=I_{W M}^{0}, R_{W}=R_{W}^{0}$.

Thus, when $\mathcal{R}_{0}=1$, the largest and only invariant set where $Q^{\prime}=0$ is also the DFE. Using LaSalle's invariance principle, one concludes that the DFE is GAS in $\Gamma_{1}$, when $\mathcal{R}_{0} \leq 1$.

Remark 3. A different proof of Theorem 1 is presented in [1], considering a different feasible set $\Gamma_{1}$.

\section{Vector, Vertical and Horizontal Transmission}

\subsection{A General Model}

The model proposed by Agusto et al.[1] and studied in Section 2 of this paper, considers vector (vector-human and human-vector) and vertical (mother-child) Zika virus transmission only. On the other hand, most other models (e. g. [2, 10, 17]) consider only vector and horizontal (human sexual) transmission. In this section, we propose a model that includes vector, vertical and horizontal transmission of Zika virus simultaneously, thus bringing together two different approaches to mathematical models of Zika virus dynamics. We show that the system in this general model has both a disease-free and an endemic equilibrium point, we study conditions under which such endemic equilibrium exists, and we provide results on global stability for both equilibria.

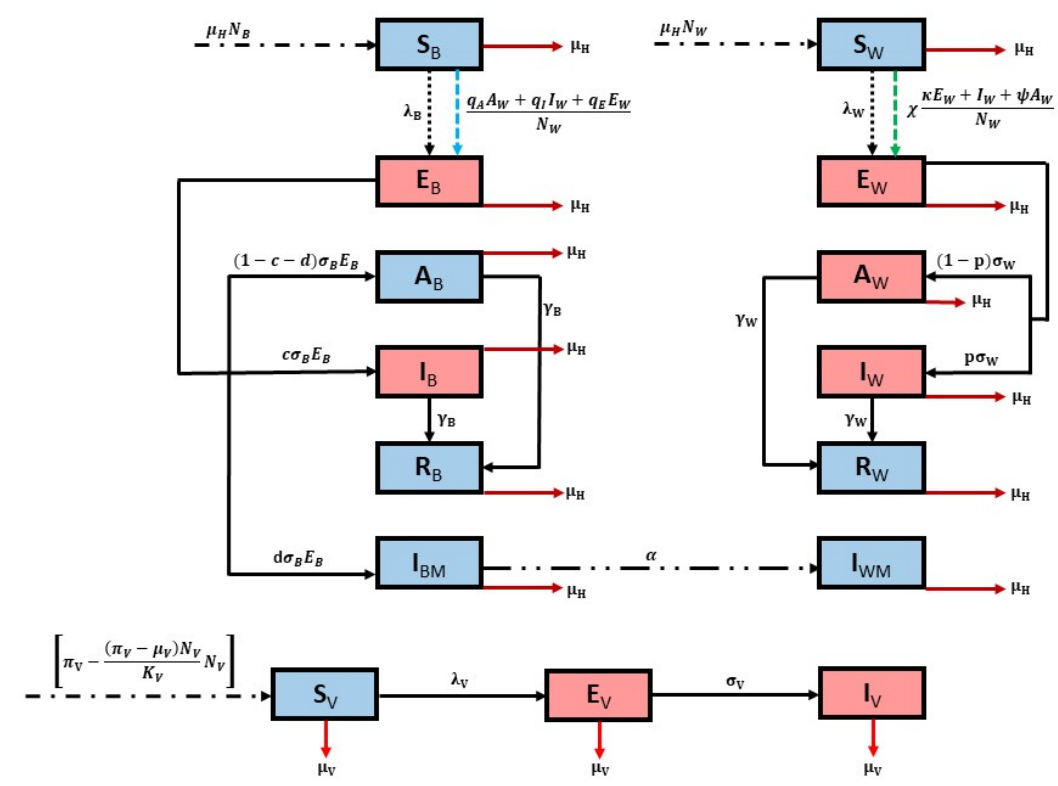

Figure 1: Adapted from Figure 1 in [1]. Blue nodes represent non-infectious compartments, while red nodes represent infectious ones. Dot-dashed arrows represent humans and mosquitoes entering the population. Black solid arrows show the progression of the disease through the system. Dotted and dashed lines show the direction of transmission. The dot-dot-dashed arrow shows the maturation of humans with microcephaly. The red arrows represent humans and mosquitoes leaving the population.

We consider the following model: 


$$
\begin{aligned}
S_{B}^{\prime} & =\mu_{H}\left(N_{B}-S_{B}\right)-\left(\frac{q_{E} E_{W}+q_{A} A_{W}+q_{I} I_{W}}{N_{W}}\right) S_{B}-\lambda_{B}\left(E_{V}, I_{V}, N_{B}\right) S_{B} \\
E_{B}^{\prime} & =\lambda_{B}\left(E_{V}, I_{V}, N_{B}\right) S_{B}+\left(\frac{q_{E} E_{W}+q_{A} A_{W}+q_{I} I_{W}}{N_{W}}\right) S_{B}-\left(\sigma_{B}+\mu_{H}\right) E_{B} \\
A_{B}^{\prime} & =(1-c-d) \sigma_{B} E_{B}-\left(\gamma_{B}+\mu_{H}\right) A_{B} \\
I_{B}^{\prime} & =c \sigma_{B} E_{B}-\left(\gamma_{B}+\mu_{H}\right) I_{B} \\
I_{B M}^{\prime} & =d \sigma_{B} E_{B}-\left(\alpha+\mu_{H}\right) I_{B M} \\
R_{B}^{\prime} & =\gamma_{B} A_{B}+\gamma_{B} I_{B}-\mu_{H} R_{B} \\
S_{W}^{\prime} & =\mu_{H}\left(N_{W}-S_{W}\right)-\chi\left(\frac{\kappa E_{W}+\psi A_{W}+I_{W}}{N_{W}}\right) S_{W}-\lambda_{W}\left(E_{V}, I_{V}, N_{W}\right) S_{W} \\
E_{W}^{\prime} & =\lambda_{W}\left(E_{V}, I_{V}, N_{W}\right) S_{W}+\chi\left(\frac{\kappa E_{W}+\psi A_{W}+I_{W}}{N_{W}}\right) S_{W}-\left(\sigma_{W}+\mu_{H}\right) E_{W} \\
A_{W}^{\prime} & =(1-p) \sigma_{W} E_{W}-\left(\gamma_{W}+\mu_{H}\right) A_{W} \\
I_{W}^{\prime} & =p \sigma_{W} E_{W}-\left(\gamma_{W}+\mu_{H}\right) I_{W} \\
I_{W M}^{\prime} & =\alpha I_{B M}-\mu_{H} I_{W M} \\
R_{W}^{\prime} & =\gamma_{W} A_{W}+\gamma_{W} I_{W}-\mu_{H} R_{W} \\
S_{V}^{\prime} & =\left(\Psi_{V}-\frac{\left(\Psi_{V}-\mu_{V}\right) N_{V}}{K_{V}}\right) N_{V}-\lambda_{V}\left(E_{B}, I_{B}, E_{W}, I_{W}, N_{B}, N_{W}\right) S_{V}-\mu_{V} S_{V} \\
E_{V}^{\prime} & =\lambda_{V}\left(E_{B}, I_{B}, E_{W}, I_{W}, N_{B}, N_{W}\right) S_{V}-\left(\mu_{V}+\sigma_{V}\right) E_{V} \\
I_{V}^{\prime} & =\sigma_{V} E_{V}-\mu_{V} I_{V},
\end{aligned}
$$

where $\lambda_{B}\left(E_{V}, I_{V}, N_{B}\right)=\frac{\eta \beta_{B} b_{V}\left(I_{V}+\theta E_{V}\right)}{N_{B}}, \lambda_{W}\left(E_{V}, I_{V}, N_{W}\right)=\frac{\beta_{W} b_{V}\left(I_{V}+\theta E_{V}\right)}{N_{W}}$, and $\lambda_{V}\left(E_{B}, I_{B}, E_{W}, I_{W}, N_{B}, N_{W}\right)=\beta_{V} b_{V}\left(\frac{\phi E_{W}+\eta \phi E_{B}+I_{W}+\eta I_{B}}{N_{W}+\eta N_{B}}\right)$.

The total human population $N_{H}=N_{B}+N_{W}$ is constant, the parameters $c$ and $d$ represent fractions of newborns who are infectious or have microcephaly respectively, and the maturation rate $\alpha$ of individuals with microcephaly is considered to be very small. Following the notation and terminology in $[2,10,16], \mu_{H}$ is the human birth and death rate, or baseline mortality of humans; the parameter $\chi$ represents the horizontal (i.e. sexual) transmission rate; $\kappa, \psi$ and $\theta$ represent transmission probabilities; $\Psi_{V}$ is the natural birth rate, and $K_{V}$ is the carrying capacity of mosquitoes. All other parameters are as in Table 1 . See $[1,10]$ for appropriate values of these parameters.

In this model, we assume babies in the womb or newly born can get the disease (first in the exposed stage) from exposed, asymptomatic and symptomatic mothers, but recovered mothers do not transmit the disease, and we assume that exposed babies progress to either asymptomatic, symptomatic or microcephalic stages. An SEI-type model is still assumed for mosquitoes, but a more general recruitment rate is used, based on a model originally proposed in [16], and considered in [2].

One can show that the feasible region for (3.1) is $\Gamma_{2}=\Gamma_{H} \times \Gamma_{V} \subset \mathbb{R}_{+}^{12} \times \mathbb{R}_{+}^{3}$, where

$$
\Gamma_{H}=\left\{\left(S_{B}, E_{B}, A_{B}, I_{B}, I_{B M}, R_{B}, S_{W}, E_{W}, A_{W}, I_{W}, I_{W M}, R_{W}\right) \in \mathbb{R}_{+}^{12}: N_{H} \leq N_{H}(0)\right\},
$$

and $\Gamma_{V}=\left\{\left(S_{V}, E_{V}, I_{V}\right) \in \mathbb{R}_{+}^{3}: N_{V} \leq K_{V}\right\}$.

The set $\Gamma_{2}$ is compact. To show that for initial nonnegative data, all variables stay nonnegative, write system (3.1) as $z_{i}^{\prime}=F_{i}(z)$, with $i=1, \ldots, 15$. If $z_{i}=0$, then clearly $F_{i}(z) \geq 0$, and hence $\Gamma_{2}$ is (positively) invariant.

System (3.1) has one disease-free equilibrium point (DFE), still denoted as $E^{0}=$ $\left(S_{B}^{0}, E_{B}^{0}, A_{B}^{0}, I_{B}^{0}, I_{B M}^{0}, R_{B}^{0}, S_{W}^{0}, E_{W}^{0}, A_{W}^{0}, I_{W}^{0}, I_{W M}^{0}, R_{W}^{0}, S_{V}^{0}, E_{V}^{0}, I_{V}^{0}\right)$, which is given by:

$$
E^{0}=\left(N_{B}, 0,0,0,0,0, N_{W}, 0,0,0,0,0, K_{V}, 0,0\right) .
$$

The system also has an endemic equilibrium point, which will be discussed in Section 3.3. 


\subsection{Global Asymptotic Stability Analysis of the DFE}

Using again the matrix-theoretic approach in [19], we split the variables into a disease compartment $x$ and a nondisease compartment $y$ :

$$
\begin{aligned}
& x=\left[E_{B}, I_{B}, E_{W}, A_{W}, I_{W}, E_{V}, I_{V}\right]^{T}, \text { and } \\
& y=\left[S_{B}, A_{B}, I_{B M}, R_{B}, S_{W}, I_{W M}, R_{W}, S_{V}\right]^{T} .
\end{aligned}
$$

Note that while the splitting of variables in Section 2.2 was made to match the work in [1], here we take a different approach: humans with microcephaly are considered to be in the nondisease compartment $y$, as they can no longer transmit the virus, and asymptomatic humans are considered in the disease compartment only if they are adults, as they can still contribute to the disease through vertical or horizontal transmission $[1,4,5,9,13]$.

Following the notation from Section 2.2, we then let

$$
\mathcal{F}=\left[\begin{array}{c}
U_{1} \\
0 \\
U_{2} \\
0 \\
0 \\
U_{3} \\
0
\end{array}\right], \quad \text { and } \quad \mathcal{V}=\left[\begin{array}{c}
\left(\sigma_{B}+\mu_{H}\right) E_{B} \\
\left(\gamma_{B}+\mu_{H}\right) I_{B}-c \sigma_{B} E_{B} \\
\left(\sigma_{W}+\mu_{H}\right) E_{W} \\
\left(\gamma_{W}+\mu_{H}\right) A_{W}-(1-p) \sigma_{W} E_{W} \\
\left(\gamma_{W}+\mu_{H}\right) I_{W}-p \sigma_{W} E_{W} \\
\left(\mu_{V}+\sigma_{V}\right) E_{V} \\
\mu_{V} I_{V}-\sigma_{V} E_{V}
\end{array}\right] \text {, }
$$

where $U_{1}=\lambda_{B}\left(E_{V}, I_{V}, N_{B}\right) S_{B}+\left(\frac{q_{E} E_{W}+q_{A} A_{W}+q_{I} I_{W}}{N_{W}}\right) S_{B}$,

$U_{2}=\lambda_{W}\left(E_{V}, I_{V}, N_{W}\right) S_{W}+\chi\left(\frac{\kappa E_{W}+I_{W}+\psi A_{W}}{N_{W}}\right) S_{W}$, and

$U_{3}=\lambda_{V}\left(E_{B}, I_{B}, E_{W}, I_{W}, N_{B}, N_{W}\right) S_{V}$. Using (2.3) as before, we get the NGM:

$$
F V^{-1}=\left[\begin{array}{ccccccc}
0 & 0 & A & B & C & D & E \\
0 & 0 & 0 & 0 & 0 & 0 & 0 \\
0 & 0 & P & G & H & I & J \\
0 & 0 & 0 & 0 & 0 & 0 & 0 \\
0 & 0 & 0 & 0 & 0 & 0 & 0 \\
K & L & M & 0 & N & 0 & 0 \\
0 & 0 & 0 & 0 & 0 & 0 & 0
\end{array}\right]
$$


where

$$
\begin{aligned}
A & =\frac{q_{E} S_{B}^{0}}{\left(\sigma_{W}+\mu_{H}\right) S_{W}^{0}}+\frac{\sigma_{W}\left(q_{A}(1-p)+q_{I} p\right) S_{B}^{0}}{\left(\sigma_{W}+\mu_{H}\right)\left(\gamma_{W}+\mu_{H}\right) S_{W}^{0}}, \\
B & =\frac{q_{A} S_{B}^{0}}{\left(\gamma_{W}+\mu_{H}\right) S_{W}^{0}}, \quad C=\frac{q_{I} S_{B}^{0}}{\left(\gamma_{W}+\mu_{H}\right) S_{W}^{0}}, \\
D & =\frac{\theta \eta \beta_{B} b_{V}}{\left(\sigma_{V}+\mu_{V}\right)}+\frac{\sigma_{V} \eta \beta_{B} b_{V}}{\mu_{V}\left(\sigma_{V}+\mu_{V}\right)}, \quad E=\frac{\eta \beta_{B} b_{V}}{\mu_{V}}, \\
P & =\frac{\chi \kappa}{\left(\sigma_{W}+\mu_{H}\right)}+\frac{\chi \sigma_{W}(p+\psi(1-p))}{\left(\sigma_{W}+\mu_{H}\right)\left(\gamma_{W}+\mu_{H}\right)}, \\
G & =\frac{\chi \psi}{\left(\gamma_{W}+\mu_{H}\right)}, \quad H=\frac{\chi}{\left(\gamma_{W}+\mu_{H}\right)}, \\
I & =\frac{\theta \beta_{W} b_{V}}{\left(\sigma_{V}+\mu_{V}\right)}+\frac{\sigma_{V} \beta_{W} b_{V}}{\mu_{V}\left(\sigma_{V}+\mu_{V}\right)}, \quad \frac{\beta_{W} b_{V}}{\mu_{V}}, \\
K & =\frac{\eta \phi \beta_{V} b_{V} K_{V}}{\left(\sigma_{B}+\mu_{H}\right)\left(S_{W}^{0}+\eta S_{B}^{0}\right)}+\frac{\eta b_{V} K_{V}}{\left(\sigma_{B}+\mu_{H}\right)\left(\gamma_{B}+\mu_{H}\right)\left(S_{W}^{0}+\eta S_{B}^{0}\right)}, \\
L & =\frac{\eta \beta_{V} b_{V} K_{V}}{\left(\gamma_{B}+\mu_{H}\right)\left(S_{W}^{0}+\eta S_{B}^{0}\right)}, \\
M & =\frac{\phi \beta_{V} b_{V} K_{V}}{\left(\sigma_{W}+\mu_{H}\right)\left(S_{W}^{0}+\eta S_{B}^{0}\right)}+\frac{\sigma_{W} p \beta_{V} b_{V} K_{V}}{\left(\sigma_{W}+\mu_{H}\right)\left(\gamma_{W}+\mu_{H}\right)\left(S_{W}^{0}+\eta S_{B}^{0}\right)}, \\
N & =\frac{\beta_{V} b_{V} K_{V}}{\left(\gamma_{W}+\mu_{H}\right)\left(S_{W}^{0}+\eta S_{B}^{0}\right)} .
\end{aligned}
$$

Unlike the original model (2.1), for this general model, the matrix $V^{-1} F$ is irreducible. Indeed,

$$
V^{-1} F=\left[\begin{array}{ccccccc}
0 & 0 & \mathcal{A}_{1} & \mathcal{A}_{2} & \mathcal{A}_{3} & \mathcal{A}_{4} & \mathcal{A}_{5} \\
0 & 0 & \mathcal{A}_{6} & \mathcal{A}_{7} & \mathcal{A}_{8} & \mathcal{A}_{9} & \mathcal{A}_{10} \\
0 & 0 & \mathcal{A}_{11} & \mathcal{A}_{12} & \mathcal{A}_{13} & \mathcal{A}_{14} & \mathcal{A}_{15} \\
0 & 0 & \mathcal{A}_{16} & \mathcal{A}_{17} & \mathcal{A}_{18} & \mathcal{A}_{19} & \mathcal{A}_{20} \\
0 & 0 & \mathcal{A}_{21} & \mathcal{A}_{22} & \mathcal{A}_{23} & \mathcal{A}_{24} & \mathcal{A}_{25} \\
\mathcal{A}_{26} & \mathcal{A}_{27} & \mathcal{A}_{28} & 0 & \mathcal{A}_{29} & 0 & 0 \\
\mathcal{A}_{30} & \mathcal{A}_{31} & \mathcal{A}_{32} & 0 & \mathcal{A}_{33} & 0 & 0
\end{array}\right],
$$

where each $\mathcal{A}_{i j}$ entry denotes a positive value. This property of irreducibility guarantees that $\mathcal{R}_{0}$ is a positive eigenvalue of $V^{-1} F$ (and of $F V^{-1}$ ), and that there exists a positive left eigenvector $w$ of $V^{-1} F$ corresponding to $\mathcal{R}_{0}$. All other assumptions $\mathcal{F}_{i}(0, y)=0, \mathcal{V}_{i}(0, y)=0, \mathcal{F}_{i}(x, y) \geq 0, \mathcal{V}_{i}(x, y) \leq 0$ when $x_{i}=0$, and $\sum_{i=1}^{7} \mathcal{V}_{i}(x, y) \geq$ 0 for $i=1, \ldots, 7$ in [19] are also satisfied.

Theorem 2. If $\mathcal{R}_{0} \leq 1$, then the disease-free equilibrium point $E^{0}$ (3.2) is globally asymptotically stable (GAS) in $\Gamma_{2}$.

Proof. As in the proof of Theorem 1, this time we have

$$
f(x, y)=\left[\begin{array}{c}
\eta \beta_{B} b_{V}\left(I_{V}+\theta E_{V}\right)\left(\frac{N_{B}-S_{B}}{N_{B}}\right)+\left(q_{E} E_{W}+q_{A} A_{W}+q_{I} I_{W}\right)\left(\frac{N_{B}-S_{B}}{N_{W}}\right) \\
0 \\
\left(\chi\left(\kappa E_{W}+I_{W}+\psi A_{W}\right)+\beta_{W} b_{V}\left(I_{V}+\theta E_{V}\right)\right)\left(\frac{N_{W}-S_{W}}{N_{W}}\right) \\
0 \\
0 \\
\beta_{V} b_{V}\left(I_{W}+\eta I_{B}+\phi E_{W}+\eta \phi E_{B}\right)\left(\frac{K_{V}-S_{V}}{N_{W}+\eta N_{B}}\right) \\
0
\end{array}\right]
$$

and therefore the condition $f(x, y) \geq 0$ holds true (this was not the case for model (2.1)). One can readily verify that the conditions $F \geq 0$ and $V^{-1} \geq 0$ are also satisfied for this new model. Then, by a direct application of Theorem 2.1 in [19], we get that $Q=\omega^{T} V^{-1} \chi$ is a Lyapunov function. 
To prove global stability, first consider $\mathcal{R}_{0}<1$. One can verify that the 8-dimensional disease-free system has the unique equilibrium point $y_{0}=\left(N_{B}, 0,0,0, N_{W}, 0,0, K_{V}\right)$, which is GAS, and that $f\left(x, y_{0}\right)=0$ in $\Gamma_{2}$. Then, by a direct application of Theorem 2.2 in [19], one concludes that if $\mathcal{R}_{0}<1$, the DFE (3.2) is GAS in $\Gamma_{2}$.

Now let $\mathcal{R}_{0}=1$. From $Q^{\prime}=0$, we get $V^{-1} f(x, y)=0$, as $\omega$ is strictly positive. It is straightforward to show that this implies $S_{B}=N_{B}, S_{W}=N_{W}$, and $S_{V}=K_{V}$, and therefore $E_{B}=A_{B}=I_{B}=I_{B M}=R_{B}=0$, and $E_{W}=A_{W}=I_{W}=I_{W M}=R_{W}=0$. Thus, when $Q^{\prime}=0$, we are left with the system

$$
\begin{aligned}
& E_{V}^{\prime}=-\left(\mu_{V}+\sigma_{V}\right) E_{V} \\
& I_{V}^{\prime}=\sigma_{V} E_{V}-\mu_{V} I_{V},
\end{aligned}
$$

which has a unique equilibrium point: $\left(E_{V}^{0}, I_{V}^{0}\right)$ - with notation as given in (3.2) - that is GAS for this system. In addition, we already have $E_{B}=E_{B}^{0}, A_{B}=A_{B}^{0}, I_{B}=I_{B}^{0}, I_{B M}=I_{B M}^{0}, R_{B}=R_{B}^{0}$, and $E_{W}=E_{W}^{0}, A_{W}=A_{B}^{0}, I_{W}=$ $I_{W}^{0}, I_{W M}=I_{W M}^{0}, R_{W}=R_{W}^{0}$. Thus, when $\mathcal{R}_{0}=1$, the largest and only invariant set where $Q^{\prime}=0$ is also the DFE. Using LaSalle's invariance principle, one concludes that the DFE is also GAS in $\Gamma_{2}$, when $\mathcal{R}_{0}=1$.

Existence of an Endemic Equilibrium. With the terminology and results established in Sections 3.1 and 3.2 above, part (2) of Theorem 2.2 in [19] guarantees the existence of an endemic equilibrium of system (3.1), when $\mathcal{R}_{0}>1$. We will denote this endemic equilibrium point as

$$
E^{\star}:=\left(S_{B}^{\star}, E_{B}^{\star}, A_{B}^{\star}, I_{B}^{\star}, I_{B M}^{\star}, R_{B}^{\star}, S_{W}^{\star}, E_{W}^{\star}, A_{W}^{\star}, I_{W}^{\star}, I_{W M}^{\star}, R_{W}^{\star}, S_{V}^{\star}, E_{V}^{\star}, I_{V}^{\star}\right) .
$$

In Theorem 2, we proved that if $\mathcal{R}_{0} \leq 1$, then the disease-free equilibrium point (DFE) is globally asymptotically stable in the given feasible region. When this condition is lost; that is, when we instead have $\mathcal{R}_{0}>1$, the DFE loses stability, and as noted above, an endemic equilibrium (EE) exists. In Theorem 3 below, we show that the condition $\mathcal{R}_{0}>1$ also implies that the EE is globally asymptotically stable.

\subsection{Global Asymptotic Stability Analysis of the EE}

To establish global stability properties of the EE (3.5), we will use a graph-theoretic method as presented in [19]. First, we briefly present some terminology and results about directed graphs and a technique for the construction of a Lyapunov function. For details, the reader is referred to [15, 19].

A pair $(i, j)$ is called an arc from vertex $i$ to vertex $j$. Given a weighted digraph $\Gamma(A)$ with $p$ vertices, the $p \times p$ weight matrix $A$ is defined with $a_{i j}>0$ equal to the weight of $\operatorname{arc}(j, i)$ if it exists, and $a_{i j}=0$ otherwise. The Laplacian $L$ of $\Gamma(A)$ is defined as

$$
l_{i j}=\left\{\begin{array}{cc}
-a_{i j}, & i \neq j, \\
\sum_{k \neq i} a_{i k}, & i=j .
\end{array}\right.
$$

Let $c_{i}$ be the cofactor of $l_{i i}$. If $\Gamma(A)$ is strongly connected, then $c_{i}>0$, for all $i=1, \ldots, p$. The following combinatorial identities are useful in finding explicit expressions for $c_{i}$ :

If $a_{i j}>0$ and the out-degree of vertex $j$ satisfies $d^{+}(j)=1$, for some $i, j$, then

$$
c_{i} a_{i j}=\sum_{k=1}^{p} c_{j} a_{j k} .
$$

If $a_{i j}>0$ and the in-degree of vertex $i$ satisfies $d^{-}(i)=1$, for some $i, j$, then

$$
c_{i} a_{i j}=\sum_{k=1}^{p} c_{k} a_{k i} .
$$

The following theorem provides a graph-theoretic technique to construct a Lyapunov function $Q$. 
Theorem 3. [15, 19] For a given open set $E \subset \mathbb{R}^{m}$, and a function $f: E \rightarrow \mathbb{R}^{m}$, consider the system

$$
\dot{z}=f(z),
$$

and assume that

(i) There exist functions $Q_{i}: E \rightarrow \mathbb{R}, G_{i j}: E \rightarrow \mathbb{R}$, and constants $a_{i j} \geq 0$ such that

$$
Q_{i}^{\prime}=\left.Q_{i}^{\prime}\right|_{(3.8)} \leq \sum_{j=1}^{p} a_{i j} G_{i j}(z), \quad \text { with } \quad z \in E, \quad i=1, \ldots, p,
$$

(ii) Each directed cycle $C$ of $\Gamma(A)$ satisfies

$$
\sum_{(s, r) \in S(C)} G_{r S}(z) \leq 0, \quad z \in E
$$

where $S(C)$ denotes the set of all arcs in $C$.

Then, there exist constants $c_{i} \geq 0, i=1, \ldots, p$ (as defined above), such that the function

$$
Q(z)=\sum_{i=1}^{p} c_{i} Q_{i}(z)
$$

satisfies $\left.Q^{\prime}\right|_{(3.8)} \leq 0$, that is, $Q(z)$ is a Lyapunov function for (3.8).

With these tools at hand, we give a result on global stability of the endemic equilibrium of system (3.1) in the interior of the feasible region $\Gamma_{2}$.

Theorem 4. If $\mathcal{R}_{0}>1$, then, the EE of system (3.1) is unique and globally asymptotically stable (GAS) in $\operatorname{int}\left(\Gamma_{2}\right)$.

Proof. Define the functions:

$$
\begin{aligned}
& Q_{1}=S_{B}-S_{B}^{\star}-S_{B}^{\star} \ln \frac{S_{B}}{S_{B}^{\star}}+E_{B}-E_{B}^{\star}-E_{B}^{\star} \ln \frac{E_{B}}{E_{B}^{\star}}, \\
& Q_{2}=I_{B}-I_{B}^{\star}-I_{B}^{\star} \ln \frac{I_{B}}{I_{B}^{\star}}, \\
& Q_{3}=S_{W}-S_{W}^{\star}-S_{W}^{\star} \ln \frac{S_{W}}{S_{W}^{\star}}+E_{W}-E_{W}^{\star}-E_{W}^{\star} \ln \frac{E_{W}}{E_{W}^{\star}}, \\
& Q_{4}=A_{W}-A_{W}^{\star}-A_{W}^{\star} \ln \frac{A_{W}}{A_{W}^{\star}}, \\
& Q_{5 a}=Q_{5 b}=Q_{5 c}=I_{W}-I_{W}^{\star}-I_{W}^{\star} \ln \frac{I_{W}}{I_{W}^{\star}}, \\
& Q_{6}=S_{V}-S_{V}^{\star}-S_{V}^{\star} \ln \frac{S_{V}}{S_{V}^{\star}}+E_{V}-E_{V}^{\star}-E_{V}^{\star} \ln \frac{E_{V}}{E_{V}^{\star}}, \\
& Q_{7}=I_{V}-I_{V}^{\star}-I_{V}^{\star} \ln \frac{I_{V}}{I_{V}^{\star}} .
\end{aligned}
$$

Using the inequality $1-x+\ln x \leq 0$, for $x>0$, differentiation yields:

$$
\begin{aligned}
Q_{1}^{\prime} \leq & q_{A} \frac{A_{W}^{\star} S_{B}^{\star}}{N_{W}^{\star}}\left(\frac{A_{W}}{A_{W}^{\star}}-\ln \frac{A_{W}}{A_{W}^{\star}}-\frac{E_{B}}{E_{B}^{\star}}+\ln \frac{E_{B}}{E_{B}^{\star}}\right) \\
& +q_{I} \frac{I_{W}^{\star} S_{B}^{\star}}{N_{W}^{\star}}\left(\frac{I_{W}}{I_{W}^{\star}}-\ln \frac{I_{W}}{I_{W}^{\star}}-\frac{E_{B}}{E_{B}^{\star}}+\ln \frac{E_{B}}{E_{B}^{\star}}\right) \\
& +q_{E} \frac{E_{W}^{\star} S_{B}^{\star}}{N_{W}^{\star}}\left(\frac{E_{W}}{E_{W}^{\star}}-\ln \frac{E_{W}}{E_{W}^{\star}}-\frac{E_{B}}{E_{B}^{\star}}+\ln \frac{E_{B}}{E_{B}^{\star}}\right)
\end{aligned}
$$




$$
\begin{aligned}
& +\eta \beta_{B} b_{V} \frac{I_{V}^{\star} S_{B}^{\star}}{N_{B}^{\star}}\left(\frac{I_{V}}{I_{V}^{\star}}-\ln \frac{I_{V}}{I_{V}^{\star}}-\frac{E_{B}}{E_{B}^{\star}}+\ln \frac{E_{B}}{E_{B}^{\star}}\right) \\
& +\eta \beta_{B} b_{V} \theta \frac{E_{V}^{\star} S_{B}^{\star}}{N_{B}^{\star}}\left(\frac{E_{V}}{E_{V}^{\star}}-\ln \frac{E_{V}}{E_{V}^{\star}}-\frac{E_{B}}{E_{B}^{\star}}+\ln \frac{E_{B}}{E_{B}^{\star}}\right) \\
& =: a_{1,4} G_{1,4}+a_{1,5 a} G_{1,5 a}+a_{1,3} G_{1,3}+a_{1,7} G_{1,7}+a_{1,6} G_{1,6} \text {. } \\
& Q_{2}^{\prime} \leq c \sigma_{B} E_{B}^{\star}\left(\frac{E_{B}}{E_{B}^{\star}}-\ln \frac{E_{B}}{E_{B}^{\star}}-\frac{I_{B}}{I_{B}^{\star}}+\ln \frac{I_{B}}{I_{B}^{\star}}\right)=: a_{2,1} G_{2,1} . \\
& Q_{3}^{\prime} \leq \beta_{W} b_{V} \frac{I_{V}^{\star} S_{W}^{\star}}{N_{W}^{\star}}\left(\frac{I_{V}}{I_{V}^{\star}}-\ln \frac{I_{V}}{I_{V}^{\star}}-\frac{E_{W}}{E_{W}^{\star}}+\ln \frac{E_{W}}{E_{W}^{\star}}\right) \\
& +\beta_{W} b_{V} \theta \frac{E_{V}^{\star} S_{W}^{\star}}{N_{W}^{\star}}\left(\frac{E_{V}}{E_{V}^{\star}}-\ln \frac{E_{V}}{E_{V}^{\star}}-\frac{E_{W}}{E_{W}^{\star}}+\ln \frac{E_{W}}{E_{W}^{\star}}\right) \\
& +\chi \frac{I_{W}^{\star} S_{W}^{\star}}{N_{W}^{\star}}\left(\frac{I_{W}}{I_{W}^{\star}}-\ln \frac{I_{W}}{I_{W}^{\star}}-\frac{E_{W}}{E_{W}^{\star}}+\ln \frac{E_{W}}{E_{W}^{\star}}\right) \\
& +\chi \psi \frac{A_{W}^{\star} S_{W}^{\star}}{N_{W}^{\star}}\left(\frac{A_{W}}{A_{W}^{\star}}-\ln \frac{A_{W}}{A_{W}^{\star}}-\frac{E_{W}}{E_{W}^{\star}}+\ln \frac{E_{W}}{E_{W}^{\star}}\right) \\
& =: a_{3,7} G_{3,7}+a_{3,6} G_{3,6}+a_{3,5 b} G_{3,5 b}+a_{3,4} G_{3,4} \text {. } \\
& Q_{4}^{\prime} \leq(1-p) \sigma_{W} E_{W}^{\star}\left(\frac{E_{W}}{E_{W}^{\star}}-\ln \frac{E_{W}}{E_{W}^{\star}}-\frac{A_{W}}{A_{W}^{\star}}+\ln \frac{A_{W}}{A_{W}^{\star}}\right)=: a_{4,3} G_{4,3} . \\
& Q_{5 a}^{\prime} \leq p \sigma_{W} E_{W}^{\star}\left(\frac{E_{W}}{E_{W}^{\star}}-\ln \frac{E_{W}}{E_{W}^{\star}}-\frac{I_{W}}{I_{W}^{\star}}+\ln \frac{I_{W}}{I_{W}^{\star}}\right)=: a_{5 a, 3} G_{5 a, 3} . \\
& Q_{5 b}^{\prime} \leq p \sigma_{W} E_{W}^{\star}\left(\frac{E_{W}}{E_{W}^{\star}}-\ln \frac{E_{W}}{E_{W}^{\star}}-\frac{I_{W}}{I_{W}^{\star}}+\ln \frac{I_{W}}{I_{W}^{\star}}\right)=: a_{5 b, 3} G_{5 b, 3} . \\
& Q_{5 c}^{\prime} \leq p \sigma_{W} E_{W}^{\star}\left(\frac{E_{W}}{E_{W}^{\star}}-\ln \frac{E_{W}}{E_{W}^{\star}}-\frac{I_{W}}{I_{W}^{\star}}+\ln \frac{I_{W}}{I_{W}^{\star}}\right)=: a_{5 c, 3} G_{5 c, 3} . \\
& Q_{6}^{\prime} \leq \beta_{V} b_{V} \phi \frac{E_{W}^{\star} S_{V}^{\star}}{N_{W}^{\star}+\eta N_{B}^{\star}}\left(\frac{E_{W}}{E_{W}^{\star}}-\ln \frac{E_{W}}{E_{W}^{\star}}-\frac{E_{V}}{E_{V}^{\star}}+\ln \frac{E_{V}}{E_{V}^{\star}}\right) \\
& +\beta_{V} b_{V} \phi \eta \frac{E_{B}^{\star} S_{V}^{\star}}{N_{W}^{\star}+\eta N_{B}^{\star}}\left(\frac{E_{B}}{E_{B}^{\star}}-\ln \frac{E_{B}}{E_{B}^{\star}}-\frac{E_{V}}{E_{V}^{\star}}+\ln \frac{E_{V}}{E_{V}^{\star}}\right) \\
& +\beta_{V} b_{V} \frac{I_{W}^{\star} S_{V}^{\star}}{N_{W}^{\star}+\eta N_{B}^{\star}}\left(\frac{I_{W}}{I_{W}^{\star}}-\ln \frac{I_{W}}{I_{W}^{\star}}-\frac{E_{V}}{E_{V}^{\star}}+\ln \frac{E_{V}}{E_{V}^{\star}}\right) \\
& +\beta_{V} b_{V} \eta \frac{I_{B}^{\star} S_{V}^{\star}}{N_{W}^{\star}+\eta N_{B}^{\star}}\left(\frac{I_{B}}{I_{B}^{\star}}-\ln \frac{I_{B}}{I_{B}^{\star}}-\frac{E_{V}}{E_{V}^{\star}}+\ln \frac{E_{V}}{E_{V}^{\star}}\right) \\
& =: a_{6,3} G_{6,3}+a_{6,1} G_{6,1}+a_{6,5 c} G_{6,5 c}+a_{6,2} G_{6,2} \text {. } \\
& Q_{7}^{\prime} \leq \sigma_{V} E_{V}^{\star}\left(\frac{E_{V}}{E_{V}^{\star}}-\ln \frac{E_{V}}{E_{V}^{\star}}-\frac{I_{V}}{I_{V}^{\star}}+\ln \frac{I_{V}}{I_{V}^{\star}}\right)=: a_{7,6} G_{7,6} .
\end{aligned}
$$

With the constants $a_{i j}$ above and $A=\left[a_{i j}\right]$, we construct the (strongly connected) directed graph $\Gamma(A)$ in Figure 2. Along each of the cycles on the graph, one can verify that $\sum G_{i j}=0$; for instance, $G_{6,1}+G_{3,6}+G_{5 a, 3}+$ $G_{1,5 a}=0, G_{2,1}+G_{6,2}+G_{1,6}=0$, and so on. Then, by Theorem 3.5 in [19], there exist constants $c_{i}$ such that $Q=\sum_{i} c_{i} Q_{i}$ is a Lyapunov function for (3.1). To find the constants $c_{i}$, we use the combinatorial identities (3.6) or (3.7); for instance, since $d^{-}(4)=1$, we use (3.7) to get $c_{4} a_{4,3}=c_{1} a_{1,4}+c_{3} a_{3,4}$. Similarly, we find that $c_{2} a_{2,1}=c_{6} a_{6,2}, \quad c_{4} a_{4,3}=c_{1} a_{1,4}+c_{3} a_{3,4}, c_{5 a} a_{5 a, 3}=c_{1} a_{1,5 a}, c_{5 b} a_{5 b, 3}=c_{3} a_{3,5 b}, c_{5 c} a_{5 c, 3}=c_{3} a_{6,5 c}$, and $c_{7} a_{7,6}=c_{1} a_{1,7}+c_{3} a_{3,7}$. With $c_{1}=c_{3}=c_{6}=1$, we get $c_{2}=\frac{\beta_{V} b_{V} \eta I_{B}^{\star} S_{V}^{\star}}{c \sigma_{B} E_{B}^{\star}\left(N_{W}^{\star}+\eta N_{B}^{\star}\right)}, \quad c_{4}=\frac{q_{A} A_{W}^{\star} S_{B}^{\star}+\chi \psi A_{W}^{\star} S_{W}^{\star}}{N_{W}^{\star}(1-p) \sigma_{W} E_{W}^{\star}}$, 


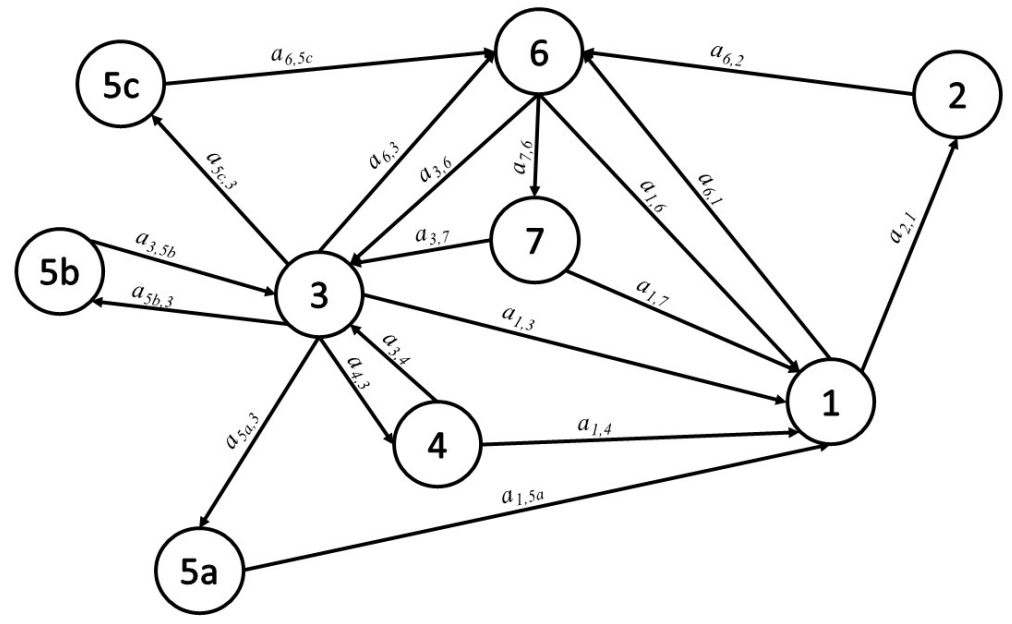

Figure 2: Digraph for model (3.1).

$c_{5 a}=\frac{q_{I} I_{W}^{\star} S_{B}^{\star}}{p \sigma_{W} E_{W}^{\star} N_{W}^{\star}}, \quad c_{5 b}=\frac{\chi I_{W}^{\star} S_{W}^{\star}}{p \sigma_{W} E_{W}^{\star} N_{W}^{\star}}, \quad c_{5 c}=\frac{\beta_{V} b_{V} I_{W}^{\star} S_{V}^{\star}}{p \sigma_{W} E_{W}^{\star}\left(N_{W}^{\star}+\eta N_{B}^{\star}\right)}$,

$c_{7}=\frac{\eta \beta_{B} b_{V} I_{V}^{\star} S_{B}^{\star} N_{W}^{\star}+\beta_{W} b_{V} I_{V}^{\star} S_{W}^{\star} N_{B}^{\star}}{\sigma_{V} E_{V}^{\star} N_{B}^{\star} N_{W}^{\star}}$.

Therefore, with the functions $Q_{i}$ and constants $c_{i}$ given above,

$$
Q=c_{1} Q_{1}+c_{2} Q_{2}+c_{3} Q_{3}+c_{4} Q_{4}+\left(c_{5 a}+c_{5 b}+c_{5 c}\right) Q_{5 c}+c_{6} Q_{6}+c_{7} Q_{7}
$$

is a Lyapunov function for (3.1). We also have:

$$
\begin{aligned}
Q^{\prime}= & \left(\frac{S_{B}-S_{B}^{\star}}{S_{B}} S_{B}^{\prime}+\frac{E_{B}-E_{B}^{\star}}{E_{B}} E_{B}^{\prime}\right)+c_{2}\left(\frac{I_{B}-I_{B}^{\star}}{I_{B}} I_{B}^{\prime}\right) \\
& +\left(\frac{S_{W}-S_{W}^{\star}}{S_{W}} S_{W}^{\prime}+\frac{E_{W}-E_{W}^{\star}}{E_{W}} E_{W}^{\prime}\right)+c_{4}\left(\frac{A_{W}-A_{W}^{\star}}{A_{W}} A_{W}^{\prime}\right) \\
& +\left(c_{5 a}+c_{5 b}+c_{5 c}\right)\left(\frac{I_{W}-I_{W}^{\star}}{I_{W}} I_{W}^{\prime}\right)+\left(\frac{S_{V}-S_{V}^{\star}}{S_{V}} S_{V}^{\prime}+\frac{E_{V}-E_{V}^{\star}}{E_{V}} E_{V}^{\prime}\right) \\
& +c_{7}\left(\frac{I_{V}-I_{V}^{\star}}{I_{V}} I_{V}^{\prime}\right) .
\end{aligned}
$$

Now we consider the set $S=\left\{x \in \mathbb{R}_{+}^{15}: Q^{\prime}=0\right\}$. When $Q^{\prime}=0$, one can readily verify that $S_{B}=S_{B}^{\star}, E_{B}=E_{B}^{\star}$, $I_{B}=I_{B}^{\star}, S_{W}=S_{W}^{\star}, E_{W}=E_{W}^{\star}, A_{W}=A_{W}^{\star}, I_{W}=I_{W}^{\star}, S_{V}=S_{V}^{\star}, E_{V}=E_{V}^{\star}$, and $I_{V}=I_{V}^{\star}$, and we are left with the system:

$$
\begin{aligned}
A_{B}^{\prime} & =(1-c-d) \sigma_{B} E_{B}^{\star}-\left(\gamma_{B}+\mu_{H}\right) A_{B} \\
I_{B M}^{\prime} & =d \sigma_{B} E_{B}^{\star}-\left(\alpha+\mu_{H}\right) I_{B M} \\
R_{B}^{\prime} & =\gamma_{B} A_{B}+\gamma_{B} I_{B}^{\star}-\mu_{H} R_{B} \\
I_{W M}^{\prime} & =\alpha I_{B M}-\mu_{H} I_{W M} \\
R_{W}^{\prime} & =\gamma_{W} A_{W}^{\star}+\gamma_{W} I_{W}^{\star}-\mu_{H} R_{W} .
\end{aligned}
$$

One can show that system (3.9) has a unique equilibrium point - see notation in (3.5): $\left(A_{B}^{\star}, I_{B M}^{\star}, R_{B}^{\star}, I_{W M}^{\star}, R_{W}^{\star}\right.$ ), and that this point is GAS for this system. Therefore, the largest and only invariant set in $S$ is the endemic equilibrium, $E^{\star}$. Using LaSalle's Invariance Principle, we conclude that the endemic equilibrium $E^{\star}$ is GAS in $\operatorname{int}\left(\Gamma_{2}\right)$, and thus unique. 


\section{Conclusions and Final Remarks}

In this article, two distinct approaches to modeling the dynamics of Zika virus have been combined into a general model. While previous work on the first approach is focused on vector and horizontal transmission $[2,10,14,17]$, a recent work [1] included vector and vertical (but not horizontal) transmission of Zika virus. In this work, the model proposed in [1] was revisited, and a global stability result of the corresponding DFE was established, under fewer restrictions. The main contribution of this work is to propose a general model of Zika virus dynamics that simultaneously includes vector, horizontal and vertical transmission, and to establish global stability results on both the DFE and the EE of this new system. These results on global stability have been established using matrix-theoretic and graph-theoretic techniques introduced in $[15,19]$, which allow the construction of appropriate Lyapunov functions. It is important to note that even though the main two theorems in [19] cannot be directly applied for the model proposed in [1], global stability of the DFE can still be obtained using the same matrix-theoretic technique.

While it is crucial to have more data to get a better biological understanding of Zika virus and to construct more accurate mathematical models, there are some possible avenues of improvement in the modeling of this mosquito-borne disease. A more accurate modeling of vertical transmission is needed, including the one through breast milk [3,5], and how the stage of the infection of the mother determines the possible stages of infection of the newborn. A better understanding and more accurate mathematical modeling of the dynamics of humans with microcephaly due to Zika infection is also needed. Some other generalizations of these models would be worth investigating, including the study of human connectivity between communities, as in $[6,12$, 18], and a consideration of the so-called "critical community size", below which the disease would probably die out [14]. Some specific heterogeneities in humans such as gender, would be worth studying. While the introduction of these new features into the current models could potentially cause losing some mathematical analytical tractability, it should bring richer dynamics, including the possible existence of some bifurcation phenomena, and a better understanding of the disease.

Acknowledgement: This work was supported in part by NSF Grant DMS 1559911

\section{References}

[1] F. B. Agusto, S. Bewick, W. F. Fagan, Mathematical model of Zika virus with vertical transmission, Infect. Disease Model., 2 (2017), 244-267.

[2] S. Bates, H. Hutson, J. Rebaza, Global Stability of Zika Virus Dynamics, J. Differ Equ Dyn Syst (2017). https://doi.org/10.1007/s12591-017-0396-0.

[3] G. Blohm, J. Lednicky, M. Marquez, S. White, J. Loeb et al. Evidence for mother-to-child transmission of Zika virus through breast milk, Clinical Infectious Diseases 66 (2018), 1120-1221.

[4] V. M. Cao-Lormeau, A. Blake, S. Mons, S. Lastere, C. Roche et al., Guillain-Barre syndrome outbreak associated with Zika virus infection in French Polynesia, The Lancet, 387 (2016), 1531-1539.

[5] M. Cavalcantia, M. Cabral-Castro, J. Goncalves, L. Santana, E. Scarlatelli, J. Peralta, Zika virus shedding in human milk during lactation: an unlikely source of infection?, Int. J. Infectious Diseases 57 (2017), 70-72.

[6] C. Cross, A. Edwards, D. Mercadante, J. Rebaza, Dynamics of a networked connectivity model of epidemics, Discr. \& Cont. Dyn. Syst., Series B, 21 (2016), 3379-3390.

[7] O. Diekmann, J. A. Heesterbeek, J. A. Metz, On the definition and the computation of the basic reproduction number ration $R_{0}$ in models for infectious diseases in heterogeneous populations, J. Math. Biol. 28 (1990), 365-382.

[8] M. R. Duffy et al. Zika virus outbreak on Yap Island, Federated States of Micronesia. New Engl. J. Med. 360 (2009), 2536-2543

[9] T. Freour, S. Mirallié, B. Hubert, C. Splingart, P. Barriere, M. Maquart, I. Leparc-Goffart, Sexual transmission of Zika virus in an entirely asymptomatic couple returning from a Zika epidemic area, Eurosurv. 21 (2016), https://doi.org/10.2807/15607917.ES.2016.21.23.30254

[10] D. Gao, Y. Lou, D. He, T. Porco, Y. Kuang, G. Chowell, S. Ruan, Prevention and control of Zika as a mosquito-borne and sexually transmitted disease: A mathematical modeling analysis, Scientific Reports, 628070 (2016).

[11] S. Garba, A. Gumel, M. Bakar, Backward bifurcations in dengue transmission dynamics, Math. Biosc. 215 (2008), 11-25. 
[12] M. Gatto, L. Mari, E. Bertuzzo, R. Casagrandi, L. Righetto, I. Rodriguez-Iturbe, A. Rinaldo, Generalized reproduction numbers and the prediction of patterns in waterborne disease, Proceed. Nat. Acad. of Scienc. 109 (2012), 1-6.

[13] B. Hughes, K. Addanki, A. Sriskanda, E. McLean, O. Bagasra, Infectivity of Immature Neurons to Zika Virus: A Link to Congenital Zika Syndrome, EBioMedicine 10 (2016), 65-70.

[14] A. Kucharski, S. Funk, R. Eggo, H. P. Mallet, W. Edmunds, A. Nilles, Transmission dynamics of Zika Virus in island populations: A modelling analysis of the 2013-14 French Polynesia outbreak, PLOS Neglected Tropical Diseases (2016), http://dx.doi.org/10.1371/journal.pntd.0004726

[15] M. Y. Li, Z. Shuai, Global stability problems for coupled systems of differential equation on networks, J. Differential Equations 248 (2010), 1-20.

[16] C. Manore, K. Hickmann, S. Xu, H. Wearing, J. Hyman Comparing dengue and chikungunya emergence and endemic transmission in A. Aegypti and A. Albopictus, J. Theor. Biology 356 (2014), 174-191.

[17] V. Moreno, B. Espinoza, D. Bicharra, S. Holecheck, C. Castillo-Chavez, Role of short-term dispersal on the dynamics of Zika virus, Infect. Disease Modeling 2(2017), 21-34.

[18] J. Rebaza, Global stability of a networked connectivity model of disease epidemics, Dyn. Cont. Discr. \&. Impuls. Syst. Series B, 23 (2016), 239-250.

[19] Z. Shuai, P. Van Den Driessche, Global stability of infectious disease models using Lyapunov functions, SIAM J. Appl. Math. 73 (2013): 1513-1532.

[20] Y. Shi, S. Li, Q. Wu, L. Sun et al. Vertical transmission of the Zika virus causes neurological disorders in mouse offspring, Scientific Reports, 8 (2018) 1-14.

[21] P. Van den Driessche, J. Watmough, Reproduction numbers and sub-threshold endemic equilibria for compartmental models of disease transmission, Math. Biosci. 180 (2002), 29-48. 